\title{
Fuzzy Logic Control for Energy Saving in Autonomous Electric Vehicles
}

\author{
A. O. A1-Jazaeri, L. Samaranayake (SMIEEE), S. Longo (MIEEE), D. J. Auger (MIEEE) \\ Centre for Automotive Engineering \\ School of Aerospace, Transport and Manufacturing, Cranfield University, United Kingdom \\ a.a.aljazaeri, 1.b.Samaranayake, s.longo,d.j.auger@cranfield.ac.uk
}

\begin{abstract}
Limited battery capacity and excessive battery dimensions have been two major limiting factors in the rapid advancement of electric vehicles. An alternative to increasing battery capacities is to use better: intelligent control techniques which save energy on-board while preserving the performance that will extend the range with the same or even smaller battery capacity and dimensions. In this paper, we present a Type-2 Fuzzy Logic Controller (Type-2 FLC) as the speed controller, acting as the Driver Model Controller (DMC) in Autonomous Electric Vehicles (AEV). The DMC is implemented using realtime control hardware and tested on a scaled down version of a back to back connected brushless DC motor setup where the actual vehicle dynamics are modelled with a Hardware-In-theLoop (HIL) system.
\end{abstract}

Using the minimization of the Integral Absolute Error (IAE) has been the control design criteria and the performance is compared against Type-1 Fuzzy Logic and Proportional Integral Derivative DMCs. Particle swarm optimization is used in the control design. Comparisons on energy consumption and maximum power demand have been carried out using HIL system for NEDC and ARTEMIS drive cycles. Experimental results show that Type-2 FLC saves energy by a substantial amount while simultaneously achieving the best IAE of the control strategies tested.

Keywords- autonomous electric vehicle, driver model controller, energy saving, hardware in the loop, Type 2 fuzzy logic controller

\section{INTRODUCTION}

With the ever decreasing fossil fuel reserves and increasing environmental awareness, considerable commitments have been made in the ground vehicle passenger transport industry to change the prime mover from internal combustion engine to electric motor. As new economies develop and emerge, urban traffic is on the increase, and there are ever more vehicles on the roads. This can manifest itself as frequent, lengthy traffic jams and there are more opportunities for risk due to weaknesses from the driver side such as negligence, tiredness, ill health, over age, etc. As a solution to this problem, autonomous vehicle options where the human driver's role gets replaced by automated control systems, have been investigated by various teams.

In a normal vehicle, the driver uses the gas pedal to set the torque reference and there is no direct feedback on the actual torque. Instead, the vehicle dynamics and road conditions result in a corresponding speed of the vehicle, which is indicated on the dash board as the feedback. Hence for the torque reference set by the driver, the feedback is the speed of the vehicle and the driver himself acts as the intelligent speed controller [1]. However in an Autonomous Electric Vehicle (AEV) [2] - [6], a Driver Model Controller (DMC) is required for this task. Depending on the objectives, different control strategies may be used for DMC [7] - [8]. For an AEV, it is important that the limited battery energy on board is optimally used while delivering performance. One measure of an autonomous vehicle's performance is its ability to follow a reference velocity profile over time. In this paper, we have defined our control objective as the minimization of the Integral Absolute Error (IAE) of the velocity compared to the reference profile.

Using Type-1 Fuzzy Logic Controller (FLC) [9] - [10] in torque control of motors in electric vehicles and industrial processes can be found in [11] -[12], where many of the systems are linear. In this study, the AEV system from the torque reference to speed feedback as shown in Fig. 1a is identified to be nonlinear having deadband and saturation, static nonlinearities, limits on rate changes as shown in Fig. $1 \mathrm{~b}$. Therefore, Type-2 FLC [13] is needed to address the nonlinerities effectively, in addition to minimizing the IAE. Type-2 Fuzzy neural networks used for trajectory control of autonomous tractor can be found in [14]. The Particle Swarm Optimization (PSO) is the optimization tool used here to find local and global optimized solutions to the control problem [15].

This paper presents the design, implementation and performance comparison of Type 2 Fuzzy Logic Controller for the DMC of an AEV, where the controller parameters are obtained online using PSO.

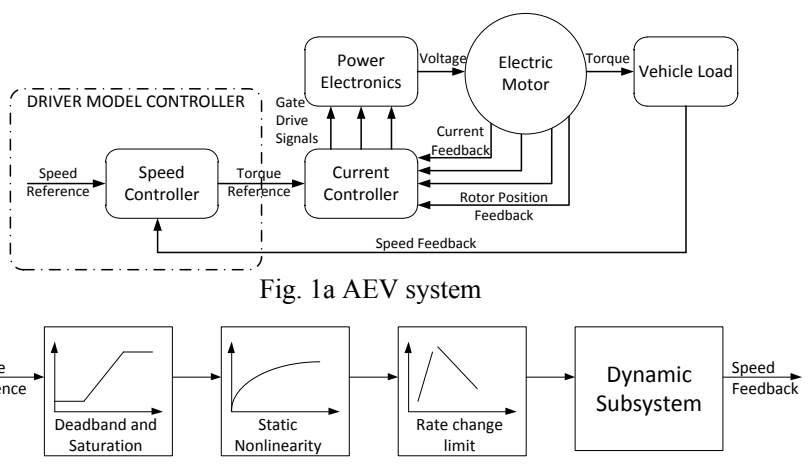

Fig. $1 \mathrm{~b}$ Nonlinear model of the system 
The paper is organized as follows: Section II is dedicated to present the design of the Type-2 FLC using Particle Swarm Optimization. Further, Type-1 FLC and PID controller designs are also outlined. Section III describes the experimental setup used in the study together with the identified nonlinear model of the system. The results are presented and discussed in the Section IV and paper is concluded with proposed future work in the Section V.

\section{INTElLigent DRIVER MODEL CONTROLleR DESIGN}

The generic block diagram for a Type-2 FLC is shown in Fig. 2. All the features are similar to Type-1 FLC except the Type Reducer block.

In the Type-2 FLC, Input 1 and Input 2 are the error and the change of error between the speed setting and the actual speed of the vehicle respectively and they can be expressed as

$$
\begin{aligned}
& e(k)=w_{\text {ref }}(k)-w(k) \\
& \Delta e(k)=e(k)-e(k-1) .
\end{aligned}
$$

The signal $u$ is the output of the controller, i.e., torque reference to the current controller in Fig. 1a. The rule base used in the FLC is shown in TABLE I, where N, P, Z, S and L stand for 'Negative', 'Positive', 'Zero', 'Small' and 'Large' respectively.

The Inference Engine, i.e, the membership functions (MF) in the Type-2 FLC have been made by double triangles (upper triangle and lower triangle) consisting of five terminal points. Hence the MF is not single valued as in the case of Type-1 FLC. In the design, five such MF triangles are used as shown in Fig. 3, where four of them are unsymmetrical and the one in the center is symmetrical about $x=0$. The higher the number of MF triangles, the more accurate the results are. However, it comes at the expense of a higher computational burden.

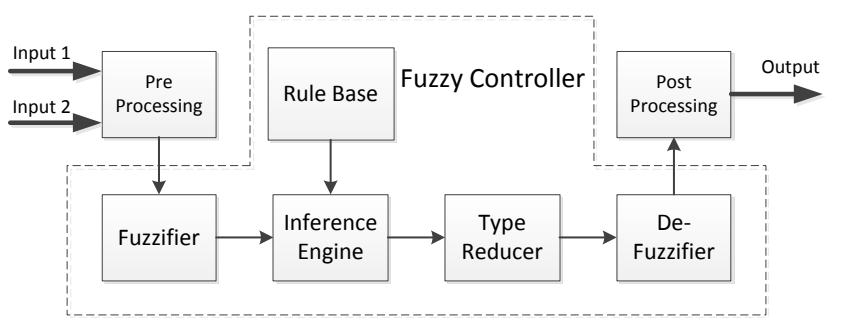

Fig. 2 Generic diagram of FLC

TABLE I: RULE BASE FOR THE TYPE-2 FLC

\begin{tabular}{|c|c|c|c|c|c|c|}
\hline \multicolumn{2}{|c|}{} & \multicolumn{5}{|c|}{$\Delta \mathbf{e}$} \\
\cline { 3 - 7 } \multicolumn{2}{|c|}{} & NL & NS & Z & PS & PL \\
\hline \multirow{4}{*}{ e } & NL & NL & NL & NL & NS & Z \\
\cline { 2 - 7 } & NS & NL & NL & NS & Z & PS \\
\cline { 2 - 7 } & Z & NL & NS & Z & PS & PL \\
\cline { 2 - 7 } & PS & NS & Z & PS & PL & PL \\
\cline { 2 - 7 } & PL & Z & PS & PL & PL & PL \\
\hline
\end{tabular}

Selecting 5 MFs result in a satisfactory computational burden without compromising the accuracy. The MF can be described as follows:

$$
\begin{aligned}
& \text { Center MF: }[-A 1,-A 2,0, A 2, A 1] \in[-1,1] \\
& \text { Upper MF: [-D, -E, -F, -G, -H, H, G, F, E, D] } \in[-1,1] \\
& \text { Lower MF: }[-E,-F,-G,-H, G,-G,-H, G, F, E] \in[-1,1]
\end{aligned}
$$

The numerical values for the Center, Upper and Lower MF are obtained by PSO. Each particle remembers its best position obtained so far $\left(p_{\text {best }}\right)$. It also receives the globally best position achieved by any particle in the population $\left(g_{\text {best }}\right)$. The updated velocity of each particle can be calculated using the present velocity and the distances from $p_{\text {best }}$ and $g_{\text {best }}$ using (4),

$v_{i, j}(k+1)=w \cdot v_{i, j}(k)+C_{1} \cdot r_{1}\left(g_{\text {best }, j}-x_{i, j}(k)\right)+C_{2} \cdot r_{2}\left(p_{\text {best }, j}-x_{i, j}(k)\right)$

where:

$v_{i, j}(k)$ is the velocity of particle $i$ in dimension $j$. The maximum values for $i$ and $j$ in this work are 80 and 10 respectively.

$x_{i, j}(k)$ is the position of particle $i$ in dimension $j$.

$C_{1}, C_{2}$ are the acceleration constants, which in this case are 1.2 and 0.12 respectively.

$w$ is the inertia weight factor which in this case is set to a global constant at 0.92 .

$r 1, r 2$ are random numbers between 0 and 1 .

This position is updated using present position and velocity as

$$
x_{i, j}(k+1)=x_{i, j}(k)+v_{i, j}(k)
$$

The initial values of swarm positions and velocities are random. The fitness function of convergence is defined as the inverse of Integrated Absolute Error (IAE) where

$$
I A E=\int_{t_{i}}^{t_{f}}|e(t)| d t
$$

and it is calculated at the end of each iteration. The boundary values for the parameters are calculated based on the minimum to maximum torque reference to the AEV system.

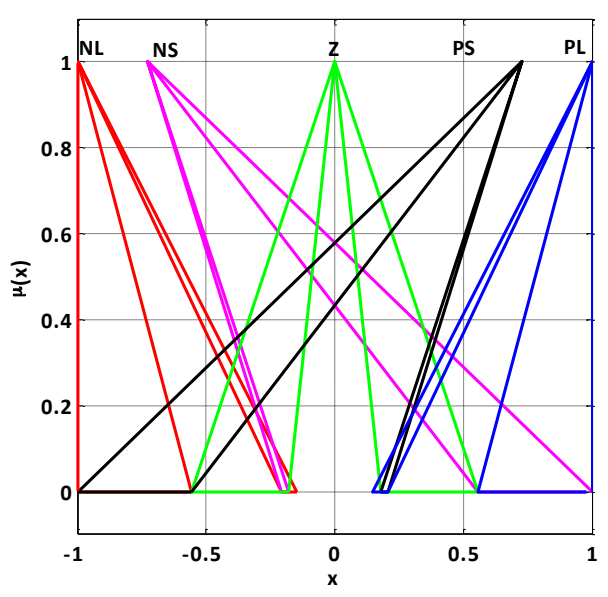

Fig. 3 MF based on PSO for Type-2 FLC 
The width of the MF, i.e., Foot of print uncertainty is varied dynamically, according to the input range. MFs with the same format as above but with different numerical values are used for Input 1, Input 2 and the Output. The 'Type Reducer' block reduces the Type- 2 sets to Type- 1 sets by Karnik-Mendel type reduction method [10]. The output of Karnik-Mendel is two folded as output left $\left(y_{l}\right)$ and output right $\left(y_{r}\right)$. Since the typereduction set is an interval Type- 2 set, the de-fuzzified output is the average of $y_{l}$ and $y_{r}$.

The Type-1 FLC is also designed using PSO. It uses the same centers of triangles of MFs as the Type-2 FLC. However, the bases of the triangles of the MFs are the algebraic averages of the upper and lower MF of the Type-2 FLC. The same rule base as in TABLE I is used here.

In the PID controller too, its parameters $k_{p}, k_{i}$ and $k_{d}$ for the Proportional, Integral and Derivative terms respectively have been designed using PSO to minimize IAE.

The minimum IAE values obtained in validating the controller design in Simulink ${ }^{\mathrm{TM}}$ simulations on the identified nonlinear AEV model are shown in TABLE II.

TABLE II: MINIMUM IAE VALUES FOR EACH CONTROLLER

\begin{tabular}{|c|c|}
\hline Controller & $\begin{array}{c}\text { Minimum IAE } \\
\text { obtained from PSO }\end{array}$ \\
\hline PID & 50.23 \\
\hline Type-1 FLC & 48.46 \\
\hline Type-2 FLC & 47.69 \\
\hline
\end{tabular}

\section{EXPERIMENTAL SETUP}

\section{A. Setup description}

The AEV powertrain experimental setup consists of the DMC (not shown), current controller, power electronics, battery, electric motor and the generator acting as the vehicle load. The block diagram is shown in Fig. 1a and the actual setup is shown in Fig. 4. For the typical four passenger electric car with moderate performance, the motor output shall be about $60 \mathrm{~kW}$. In the experimental setup we use a scaled down version of motor with $5 \mathrm{~kW}$ output power with $60 \mathrm{~V}$ at the DC link voltage for the power electronics from the battery bank.

The shaft of the brushless DC (BLDC) motor is back-toback connected to an identical BLDC motor, which acts as a generator (Fig. 4a). The generator is used as the vehicle load acting on the motor which mimics the vehicle dynamics and other resistive loads. The BLDC generator receives the vehicle dynamic signals from Hardware-In-the-Loop system implemented using dSPACE ${ }^{\mathrm{TM}}$ system (Fig. 4b).

It consists of DS1006 processor with dSPACE 2013b real time operating system, DS2202 input-output card interfaced to the user through ControlDesk ${ }^{\mathrm{TM}} 5.1$ proprietary software platform. In the experiments, two standard drive cycles, i.e. New European Drive Cycle (NEDC) which is more urban centric but with little practical relevance, and combined Assessment and Reliability of Transport Emission Models and Inventory Systems (ARTEMIS), which is motorway centric [16], are used.

\section{B. Model of the system}

Prior to the design of the Driver Model Controller, in order to model and understand the system which will support the controller design, a System Identification experiment has been carried out for the system from the Torque Reference to Speed Feedback in the block diagram shown in Fig. 1a. It reveals that the system is nonlinear. The nonlinearities have been identified as dead-band, saturation, static nonlinearity and limitations on rate changes as shown in Fig. 1b. Generally these nonlinearities come from

1. Cogging torque in the BLDC motor and generator,

2. Use of Hall sensor position output to calculate the speed,

3. Hysteresis comparators in the current controller.

The dynamic subsystem as shown in Fig. 1b, which consists of the linear part of the nonlinear system is identified using least squares estimation method, and the transfer function is given in (6).

$$
G(s)=(-0.45 s+37.59) /\left(s^{2}+9.92 s+37.78\right)
$$

As is observed in (6), it is a non-minimum phase system, which limits the speed of operation. A comparison of the output of the identified model and the actual system is shown in Fig. 5. This model is used in the Driver Model Controller design validation in simulation, using Type-2 FLC, Type-1 FLC and PID controller prior to their hardware implementations.

The validated controllers are implemented in HIL. Here the $\mathrm{MATLAB}^{\mathrm{TM}} . \mathrm{m}$ codes are converted into $\mathrm{C}$ language code compatible to dSPACE ${ }^{\mathrm{TM}}$ using its proprietary compiling and building tools and were implemented in $\mathrm{dSPACE}^{\mathrm{TM}}$ hardware.

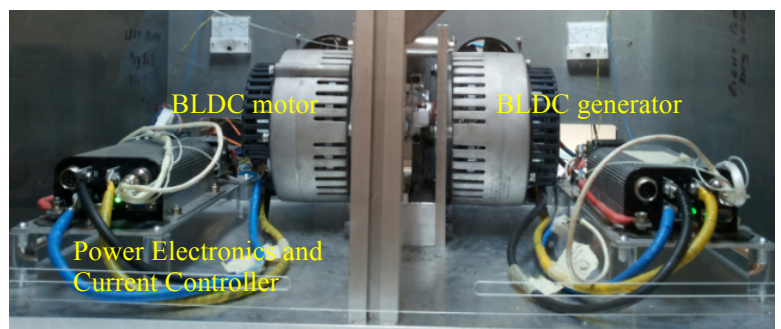

Fig. 4a Back to back connected motor generator combination

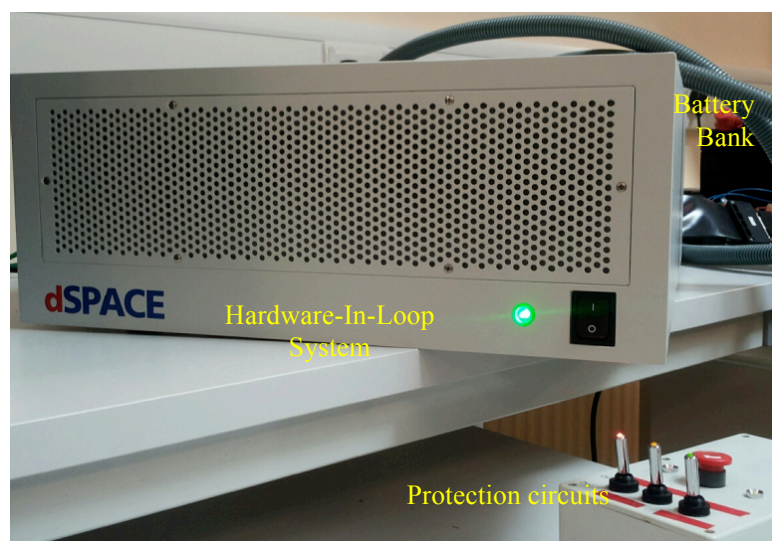

Fig. 4b dSPACE ${ }^{\mathrm{TM}}$ HIL system 

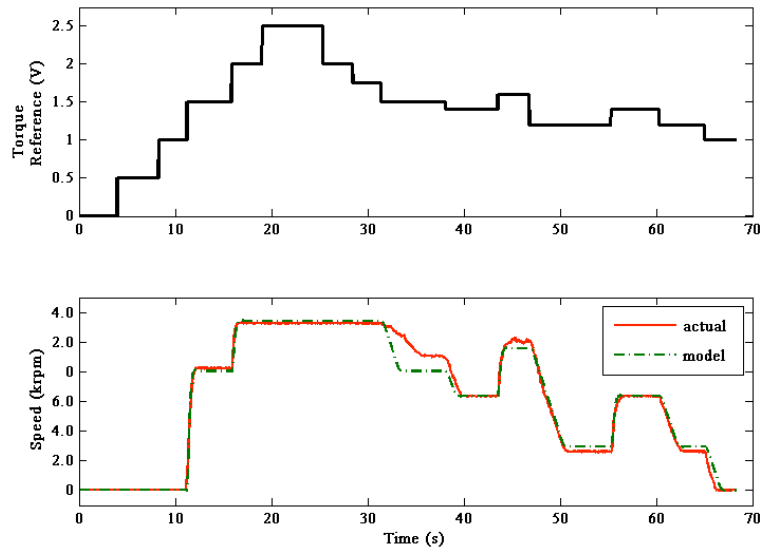

Fig. 5 Comparison of the identified model output with the actual output.

\section{RESULTS AND DISCUSSION}

\section{A. Experiment sets}

The DMC performance is investigated for
a) Transient response
b) Disturbance rejection
c) Energy demand and Peak Power demand.

For case $a$ ), a step input is used with constant torque demand, and for case $b$ ) constant and random disturbances are applied through the generator using HIL, which corresponds to friction, aerodynamic, inertia loads and other disturbances on the AEV. The case $c$ ) is divided in to two cases as c) $i$. Using NEDC and c) ii. Using combined ARTEMIS drive cycle.

The respective torque demands are derived from the drive cycles using the vehicle dynamics and applied through the generator using the HIL system.

Compared to NEDC where only $42 \%$ of the time the speed is above the average speed, in the combined ARTEMIS drive cycle, more than $65 \%$ of the duration, the speed is above the average. The normalized average speeds of the two drive cycles are 0.27 and 0.66 respectively.

\section{B. CASE (a): Transient response}

A step input is applied as the reference input to the Driver Model Controllers and their responses on constant load torque are shown in Fig. 6. As observed in TABLE III, the Type-2 FLC results in the minimum IAE. This is in agreement with the simulation results presented in TABLE II, where Type-2 FLC results in the lowest IAE. In addition, it results in the lowest percentage overshoot $\left(M_{P}\right)$, rise time $\left(t_{r}\right)$ and settling time $\left(t_{s}\right)$ as additional advantages, which makes it more suitable for DMC in an AEV preserving energy during transients.

TABLE III: DMC PERFORMANCE COMPARISON ON NO LOAD.

\begin{tabular}{|c|c|c|c|c|}
\hline Controller & $\boldsymbol{t}_{\boldsymbol{r}}(\mathbf{s})$ & $\boldsymbol{t}_{\boldsymbol{s}}(\mathbf{s})$ & $\boldsymbol{M}_{\boldsymbol{P}} \mathbf{( \% )}$ & IAE \\
\hline PID & 2.85 & 4.44 & 0.59 & 51.30 \\
\hline Type-1FLC & 1.17 & 2.22 & 0.58 & 50.76 \\
\hline Type-2FLC & 0.56 & 0.85 & 0.20 & 48.93 \\
\hline
\end{tabular}

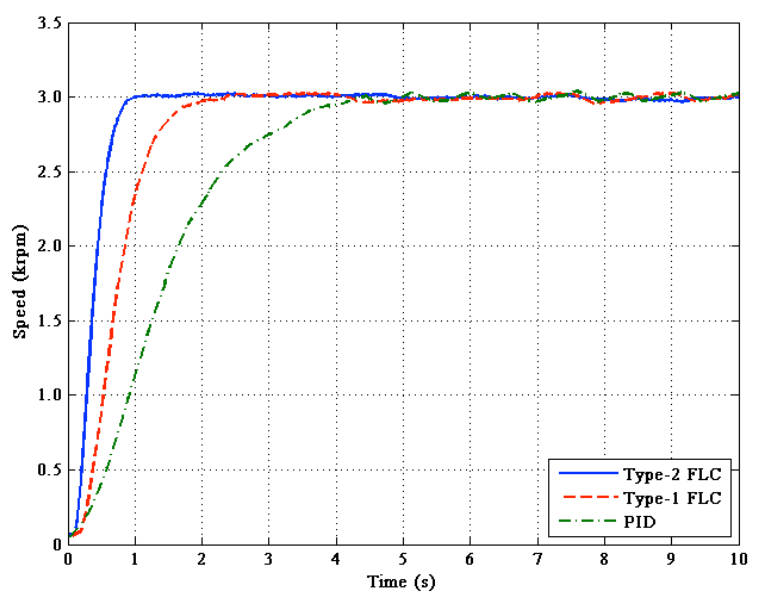

Fig. 6 Transient response with reference speed is set to $3000 \mathrm{rpm}$.

\section{CASE (b): Disturbance Rejection}

In order to compare the disturbance rejection capability of the DMC, two types of loads are used which are enforced simultaneously. They are constant loads mimicking the resistive forces and sudden disturbances mimicking road conditions. In this case, while the AEV is moving at 2000 rpm motor speed, under constant loading conditions, a sudden disturbance is applied at time $17.8 \mathrm{~s}$. The disturbance rejection response is shown in Fig. 7 and the performance comparison results, i.e., percentage overshoot $\left(M_{P}\right)$, rise time $\left(t_{r}\right)$ and settling time $\left(t_{s}\right)$, of the controllers are shown in TABLE IV.

For energy saving, fast but critically damped responses are preferred. As can be observed, in recovering from a sudden disturbance, DMC with PID takes substantially longer time with local stabilizations due to nonlinearities inherent in the system. The Type-1 FLC copes up with this situation better than PID as it recovers relatively fast. Compared to the other two controllers, Type-2 FLC is observed to be the best because it recovers faster with no overshoot. It may be due to the fact that foot of print uncertainty of $\mathrm{MF}$ is varied dynamically to address the nonlinearities.

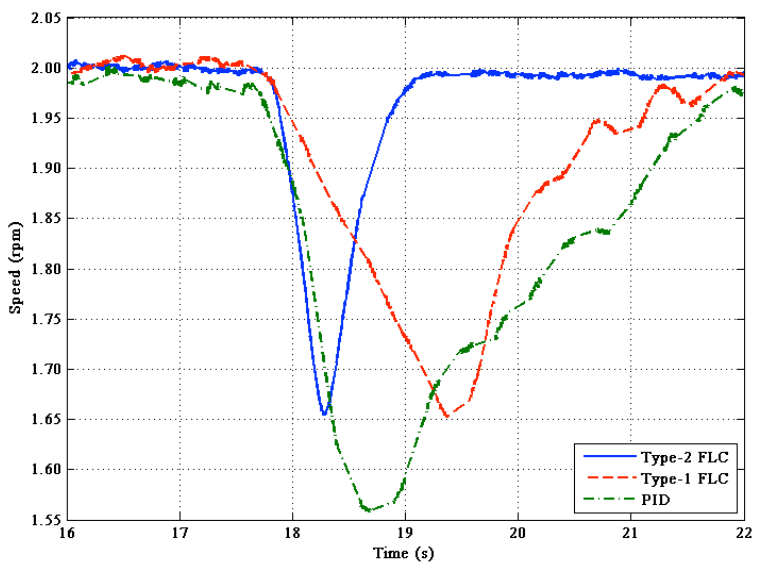

Fig. 7 Comparison of disturbance rejection responses of controllers. 
TABLE IV: COMPARISON IN DISTURBANCE REJECTION.

\begin{tabular}{|c|c|c|c|c|}
\hline Controller & $\mathbf{t}_{\mathbf{r}}(\mathbf{s})$ & $\mathbf{t}_{\mathbf{s}}(\mathbf{s})$ & $\mathbf{M}_{\mathbf{P}} \mathbf{( \% )}$ & $\mathbf{I A E}$ \\
\hline PID & 3.20 & 5.23 & 2.85 & 8.25 \\
\hline Type-1FLC & 2.23 & 2.35 & 0.60 & 8.20 \\
\hline Type-2FLC & 0.80 & 1.12 & 0.20 & 7.64 \\
\hline
\end{tabular}

\section{CASE (c) i: Energy demand and peak power demand} under NEDC

Fig. 8 shows the performance of Driver Model Controllers under NEDC. The TABLE $\mathrm{V}$ shows the maximum power demand and the total energy consumed under each DMC when the AEV is following NEDC. The instantaneous input power demand when running NEDC is shown in Fig. 9, which is obtained by taking the product of the instantaneous current to the BLDC motor and the instantaneous supply voltage.

The results in TABLE V show that Type-2 FLC has $13.7 \%$ less peak power demand as compared to that of PID and $12.9 \%$ less compared to Type-1 FLC. The energy consumption during the NEDC is also $35.0 \%$ less in Type-2 FLC compared to PID and $18.1 \%$ less than Type-1 FLC.

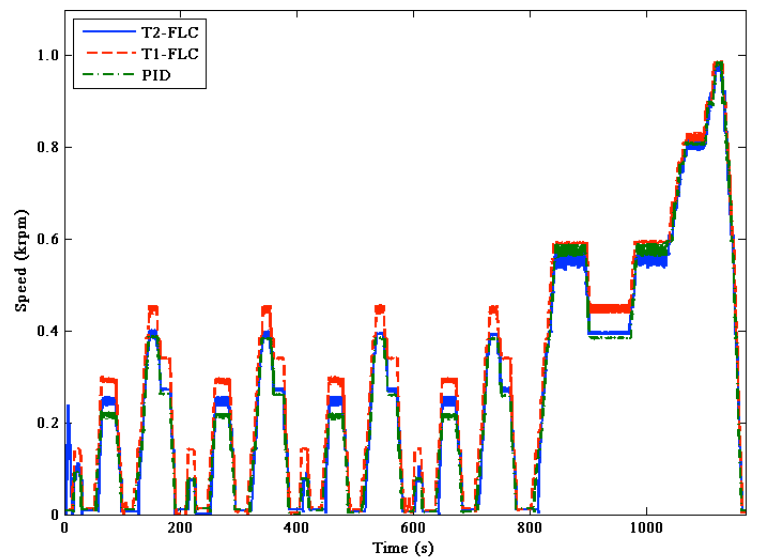

Fig. 8 Speed of motor with DMCs reference set to NEDC.

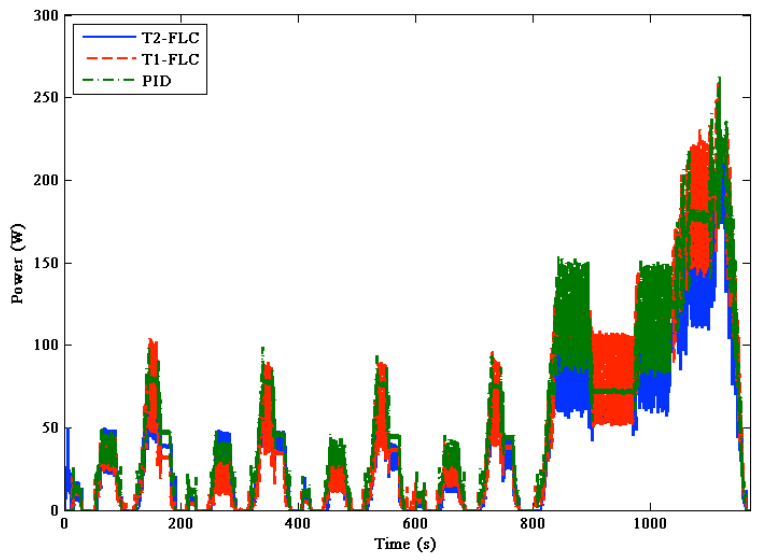

Fig. 9 Input power demand under the same vehicle load torque with different DMCs when using NEDC.
TABLE V: MAXIMUM POWER DEMAND \& TOTAL ENERGY CONSUMPTION WHILE ON NEDC

\begin{tabular}{|c|c|c|}
\hline Controller & $\begin{array}{c}\text { Maximum Power } \\
\text { Demand (W) }\end{array}$ & $\begin{array}{c}\text { Total Energy } \\
\text { consumed (kJ) }\end{array}$ \\
\hline PID & 262.35 & 30.61 \\
\hline Type-1 FLC & 259.83 & 24.30 \\
\hline Type-2 FLC & 226.41 & 19.90 \\
\hline
\end{tabular}

\section{E. CASE (c) ii: Energy demand and peak power demand under combined ARTEMIS drive cycle}

Fig. 10 shows the performance of Driver Model Controllers under ARTEMIS drive cycle. The corresponding instantaneous input power demand is shown in Fig. 11. In TABLE VI, it is observed that the Type- 2 FLC has $31.7 \%$ less peak power demand as compared to PID and 43.2\% less compared to Type-1 FLC. The energy consumption during the combined ARTEMIS drive cycle is also $28.5 \%$ less in Type-2 FLC as compared to PID and $38.1 \%$ less than Type- 1 FLC.

The vehicle load torque applied on the motor through generator during each drive cycle using the HIL system in the cases c) $i$ and $c$ ) $i i$ are in shown in Fig. 12.

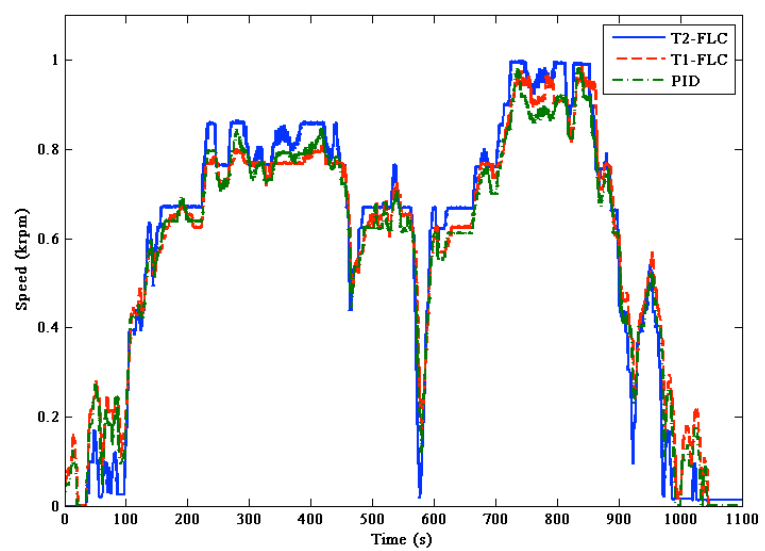

Fig. 10 Combined ARTEMIS drive cycle using each Driver Model Controller.

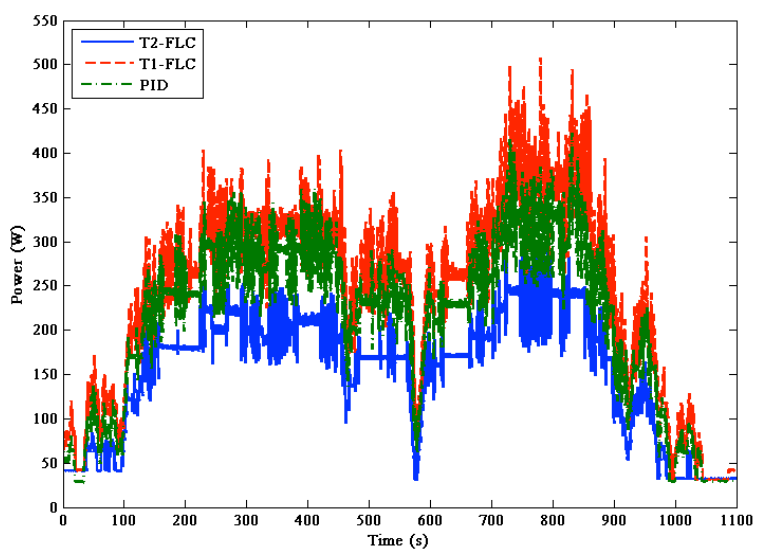

Fig. 11 Input power demand under the same vehicle load torque with different DMCs when using combined ARTEMIS drive cycle. 
TABLE VI: MAXIMUM POWER DEMAND \& TOTAL ENERGY CONSUMPTION IN COMBINED ARTEMIS DRIVE CYCLE

\begin{tabular}{|c|c|c|}
\hline Controller & $\begin{array}{c}\text { Maximum Power } \\
\text { Demand (W) }\end{array}$ & $\begin{array}{c}\text { Energy consumed } \\
(\mathbf{k J})\end{array}$ \\
\hline PID & 422.09 & 90.52 \\
\hline Type-1 FLC & 507.14 & 104.67 \\
\hline Type-2 FLC & 288.10 & 64.76 \\
\hline
\end{tabular}

It is important to note that under both drive cycles Type2 FLC out performs the reference PID and Type-1 FLC because the nonlinearities identified in the AEV system are addressed through its double triangle membership functions. It is interesting to note that Type-1 FLC demands less peak power and total energy compared to PID when using NEDC. However, the response is opposite when using combined ARTEMIS drive cycle.

The reason is that they both do not address the nonlinearities effectively and the latter nonlinearities in the system are triggered differently under the two drive cycles. Since NEDC is an urban drive cycle, its average speed is lower ( 0.27 normalized) and $58 \%$ of the duration of the cycle, the speed is below the average. On the other hand, the combined ARTEMIS being a drive cycle representing mainly the motorway driving, has a relatively higher average speed ( 0.66 normalized) and more interestingly, $65 \%$ of the time its speed is above the latter higher average. As a result, the nonlinearities excited in the two drive cycles are different, and Type-1 FLC and PID do not cater them effectively and therefore the system responds differently.

\section{CONCLUSIONS}

In both the drive cycles, while resulting in the minimum integral of the absolute error, Type-2 FLC demands significantly lower peak power (13.7\% for NEDC and $31.7 \%$ in ARTEMIS) and less total energy (35\% for NEDC and $28.5 \%$ in ARTEMIS) from the battery during the drive cycle as compared to PID controller. This will result in a substantial increase in the range of the autonomous electric vehicle and/or a smaller and lighter battery pack without compromising performance. Further, the Type-2 FLC experimentally proved to be faster in transients and disturbance rejection than the other control designs considered in the nonlinear autonomous electric vehicle model.

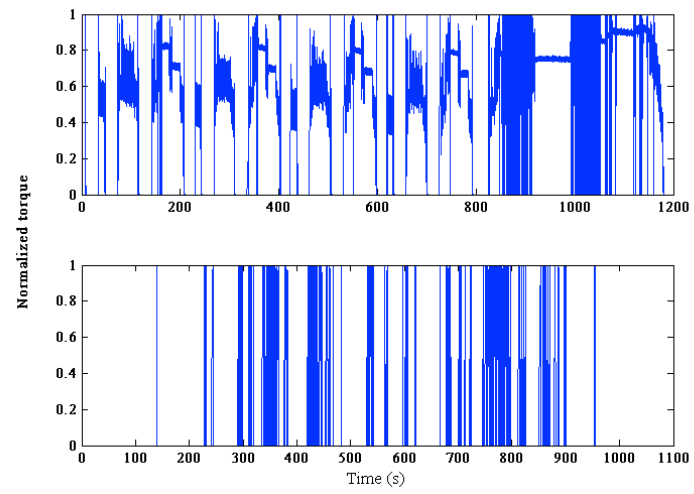

Fig. 12 External torque applied during NEDC (upper) and ARTEMIS drive cycle (lower) using HIL system.

\section{ACKNOWLEDGMENT}

Authors wish to acknowledge the financial support from the "Developing FUTURE Vehicles" research project (EPSRC grant number: EP/I038586/1).

\section{REFERENCES}

[1] Xiaohong Nian; Fei Peng; Hang Zhang, "Regenerative Braking System of Electric Vehicle Driven by Brushless DC Motor," Industrial Electronics, IEEE Transactions on, vol.61, no.10, pp.5798,5808, Oct. 2014

[2] Moriwaki, K., "Mathematical modeling and control of an autonomous electric vehicle for navigation and guidance," Electric Vehicle Conference (IEVC), 2012 IEEE International, vol., no., pp.1,8, 4-8 March 2012

[3] Voncilă, I.; Voncilă, E.; Fetecău, G., "Analysis of the stability of synchronous motor - Generator unit used in propulsion systems of autonomous electric vehicles," Electrical and Electronics Engineering (ISEEE), 2010 3rd International Symposium on, vol., no., pp.143,148, 16-18 Sept. 2010

[4] Youjun Choi; Daejun Kang; Sunjong Lee; Youngmin Kim, "The autonomous platoon driving system of the on line electric vehicle," ICCAS-SICE, 2009, vol., no., pp.3423,3426, 18-21 Aug. 2009

[5] HuiSuo; Jiafu Wan; Di Li; CaifengZou, "Energy Management Framework Designed for Autonomous Electric Vehicle with Sensor Networks Navigation," Computer and Information Technology (CIT), 2012 IEEE 12th International Conference on , vol., no., pp.914,920, 2729 Oct. 2012

[6] Silva, M.; Garrote, L.; Moita, F.; Martins, M.; Nunes, U., "Autonomous electric vehicle: Steering and path-following control systems," Electrotechnical Conference (MELECON), 2012 16th IEEE Mediterranean, vol., no., pp.442,445, 25-28 March 2012

[7] Houacine, K.; Mellah, R.; Guermah, S.; Charif, M., "Neural fuzzy control of driving wheels for electric vehicle," Systems and Control (ICSC), 2013 3rd International Conference on , vol., no., pp.25,30, 2931 Oct. 2013

[8] Tao Wang; Cassandras, C.G., "Optimal motion control for energy-aware electric vehicles," Control Applications (CCA), 2013 IEEE International Conference on, vol., no., pp.388,393, 28-30 Aug. 2013

[9] Zadeh, L.A. "Fuzzy Sets", Elsevier, Information and Control, vol 8, issue 3, pp 338-353June 1965

[10] Mendel, J. M., "Rule-Based Fuzzy Logic Systems: Introduction and New Directions" Prentice-Hall, NJ, 2001

[11] Rebeiro, R.S.; Uddin, M.N., "Performance Analysis of an FLC-Based Online Adaptation of Both Hysteresis and PI Controllers for IPMSM Drive," Industry Applications, IEEE Transactions on , vol.48, no.1, pp.12,19, Jan.-Feb. 2012

[12] Rubaai, A.; Jerry, J.; Smith, S.T., "Performance Evaluation of Fuzzy Switching Position Controller for Automation and Process Industry Control," Industry Applications, IEEE Transactions on , vol.47, no.5, pp.2274,2282, Sept.-Oct. 2011

[13] Patricia, M; Oscar, C., "Recent Advances in Interval Type-2 Fuzzy Systems" Springer Heidelberg New York Dordrecht London, 2012

[14] Kayacan, E.; Kayacan, E.; Ramon, H.; Kaynak, O.; Saeys, W., "Towards Agrobots: Trajectory Control of an Autonomous Tractor Using Type-2 Fuzzy Logic Controllers," Mechatronics, IEEE/ASME Transactions on , vol.PP, no.99, pp. 1,12

[15] Zwe-Lee Gaing, "A particle swarm optimization approach for optimum design of PID controller in AVR system," Energy Conversion, IEEE Transactions on , vol.19, no.2, pp.384,391, June 2004

[16] Barlow, T.J.; Latham, S.; McCrae, I.S.; Boulter, P.G.; “A reference book of driving cycles for use in the measurement of road vehicle emissions" available online at https:/www.gov.uk/government/uploads/system/uploads/attachment_dat a/file/4247/ppr-354.pdf 
2015-03-06

Fuzzy logic control for energy saving in autonomous electric vehicles

\author{
Al-Jazaeri, Ahmed O.
}

IEEE

A. O. Al-Jazaeri, L. Samaranayake, S. Longo and D. J. Auger. Fuzzy logic control for energy saving in autonomous electric vehicles. Proceedings of the 2014 IEEE International Electric Vehicle Conference (IEVC), 17-19 September 2014, Florence, Italy. pp1-6 http://dx.doi.org/10.1109/IEVC.2014.7056100

Downloaded from Cranfield Library Services E-Repository 